\title{
Simulation of Droplet Breakup and Evolving Rheology in a High Shear Mixer
}

\author{
Vipin Michael $^{1}$, Robert Prosser ${ }^{1}$, Adam Kowalksi \\ ${ }^{1}$ School of Mechanical, Aerospace and Civil Engineering, University of Manchester \\ Manchester, UK, M13 9PL \\ vipin.michael@manchester.ac.uk; robert.prosser@manchester.ac.uk \\ ${ }^{2}$ Unilever Research and Development, Port Sunlight Laboratory \\ Quarry Road East, Bebington, Wirral, UK, CH63 3JW \\ adam.kowalksi@unilever.com
}

\section{Extended Abstract}

High shear in-line rotor/stator mixers are widely used in industry for the manufacture of emulsion based personal care products. The properties of these formulated emulsions are process sensitive, with droplet size and viscosity affecting the physical stability and consumer appeal of the final product. Emulsion characterisation is essential for quality control, storage stability and the prediction of flow behaviour. However, industrial practice is still largely based on experimental results and empirical rules, as the understanding of droplet breakup mechanisms and processing in these devices is limited.

Increasingly, simulation is playing a role in developing an understanding of the interplay between detailed dynamics and the evolution of the emulsion. Recent simulations of droplet dispersions in a Silverson 150/250MS rotor/stator mixer have focussed on low phase volume emulsions on which, the emergent rheology is only weakly dependent [1]. In concentrated emulsions, rheological behaviour varies with the droplet statistics. Recently Baldyga et al. [2] have presented a model linking droplet size distribution to emulsion viscosity thereby allowing the simulation of evolving rheology in such mixers.

This paper describes recent efforts to couple Computational Fluid Dynamics (CFD) to Population Balance Modelling (PBM), in order to simulate the processing of concentrated oil/water emulsions. Results of transient simulations using a sliding mesh method are presented. Pressure, velocities and turbulence quantities are calculated using a rotation/curvature compensated eddy-viscosity turbulence model. Population balance equations for droplet dispersion are solved using the quadrature method of moments (QMOM) [3]. Breakage models based on the multi-fractal theory of intermittent turbulence [4] have been modified to include the effect of turbulent shear and close packing of droplets at high phase volume. The mean droplet size (d) of the emulsion after a single pass through the mixer is shown to decrease with rotor speed $(\mathrm{N})$ as $\mathrm{d} \sim$ $\mathrm{N}^{-0.821}$. Drop size distribution of the emulsion after processing show that a large number of smaller and more homogenous drops are obtained at higher rotor speeds. Flow curves characterising rheology of the emulsion after processing at different rotor speeds were obtained showing shear thinning behaviour and viscosity build with decreasing droplet size.

\section{References}

[1] M. Jasińska, J. Bałdyga, S. Hall, and A. W. Pacek, "Dispersion of oil droplets in rotor-stator mixers: Experimental investigations and modeling," Chem. Eng. Process. Process Intensif., vol. 84, pp. 45-53, 2014.

[2] J. Baldyga, M. Jasinska, and A. J. Kowalski, "Effect of rheology of dense emulsions on the flow structure in agitated systems," Chem. Eng. Res. Des., vol. 108, pp. 3-12, 2016.

[3] R. McGraw, "Description of Aerosol Dynamics by the Quadrature Method of Moments," Aerosol Sci. Technol., vol. 27, no. 2, pp. 255-265, 1997.

[4] J. Bałdyga and W. Podgorska, "Drop break-up in intermittent turbulence: Maximum stable and transient sizes of drops," Can. J. Chem. Eng., vol. 76, no. 1, pp. 456-470, 1998. 